\title{
Trapped ion quantum computation with transverse phonon modes
}

\author{
Shi-Liang Zhu, C. Monroe, and L.-M. Duan \\ FOCUS Center and MCTP, Department of Physics, University of Michigan, Ann Arbor, MI 48109
}

\begin{abstract}
We propose a scheme to implement quantum gates on any pair of trapped ions immersed in a large linear crystal, using interaction mediated by the transverse phonon modes. Compared with the conventional approaches based on the longitudinal phonon modes, this scheme is much less sensitive to ion heating and thermal motion outside of the Lamb-Dicke limit thanks to the stronger confinement in the transverse direction. The cost for such a gain is only a moderate increase of the laser power to achieve the same gate speed. We also show how to realize arbitrary-speed quantum gates with transverse phonon modes based on simple shaping of the laser pulses.
\end{abstract}

PACS numbers: 03.67.Lx, 32.80.Qk, 03.67.Pp

Trapped ions have been demonstrated as one of the most promising systems for implementation of quantum computation. Different theoretical schemes have been proposed for quantum gate operations [1, 2, 3, 4, 5, 6, 7], and many building blocks of quantum computing have been experimentally demonstrated 8, 9, 10, 11, 12, 13, 14, 15]. In an ion trap quantum computer, entangling gates between different ions are mediated through phonons in the collective ion motion. In all previous gate schemes [2, 3, 4, 5, 6, 7], the longitudinal phonon (LP) modes are exploited by kicking the ions along the axial direction of a linear trap.

In this work, we propose to use the transverse phonon (TP) modes for gate operations. Compared with the conventional schemes (hereafter referred to as LP gates), gates involving TP modes (TP gates) have the following distinctive features: First, due to the strong confinement in the transverse direction, the TP gate is much less sensitive to ion heating and thermal motion. Even if the axial ion oscillation amplitude is significantly greater than the optical wavelength (outside of the Lamb-Dicke regime), high-fidelity gates through the TP modes are still possible. If $\beta$ denotes the ratio of the center-of-mass (CM) trap frequencies for the transverse and the longitudinal directions ( $\beta \gg 1$ in typical experiments), we show that gate infidelity due to thermal ion motion is reduced by a factor ranging from $\beta^{4}$ to $\beta^{6}$, depending on details of the initial ion temperature and the heating mechanism. This improvement may be particularly significant for a system of many ions or for ions confined in a microtrap [16], where ion heating and thermal motion may dominate gate erors. Second, the cost of using the TP modes is moderate, even though it is more difficult to excite the TP modes due to their strong confinement. For TP gates to have the same speed as LP gates, the intensity of the driving laser needs only to be increased by a factor of $\sqrt{\beta / 2}$, a small factor when compared with the improvement in gate fidelity. Finally, we show that TP quantum gates can be operated with arbitrary speeds. Although the frequency splitting of the TP modes is significantly smaller than that of the LP modes, this does not impose any limit to the gate speed. High-fidelity fast TP gates are still possible through control of a simple sequence of laser pulses, which typically involves excitation of many TP modes.

Transverse phonon (TP) modes. To design TP quantum gates, we first describe the structure of the TP modes. We consider a system of $N$ ions confined in a linear trap. The phonon modes are obtained through diagonalization of the Hamiltonian for the ion external motion [17. The ion motional Hamiltonian has the form $H_{0}=\sum_{\xi} \sum_{j=1}^{N} \frac{p_{\xi, j}^{2}}{2 M}+V$, where $M$ is the ion mass, and $p_{\xi, j}$ is the momentum operator of $j$ th ion along the direction $\xi(\xi=x, y, z)$. The potential $V=\frac{M}{2} \sum_{j=1}^{N}\left(\omega_{x}^{2} x_{j}^{2}+\omega_{y}^{2} y_{j}^{2}+\omega_{z}^{2} z_{j}^{2}\right)+\sum_{n, j} \frac{e^{2}}{4 \pi \epsilon_{0} r_{n j}}$ accounts for the Coulomb interaction between the ions as well as the external trapping potential, where $r_{n j}$ denotes the distance between ions $n$ and $j$, and $\omega_{\xi}$ is the CM trap frequency along the direction $\xi$. Typically, $\omega_{x} \sim \omega_{y} \gg \omega_{z}$, and one has a linear geometry with an ion chain along the $\mathbf{z}$ axis when $\omega_{x, y} / \omega_{z}$ is larger than the critical ratio about $0.73 N^{0.86}[18]$. The equilibrium positions $z_{n}^{0}\left(x_{n}^{0}=y_{n}^{0}=0\right)$ of the ions are determined by the condition $\left[\partial V / \partial z_{n}\right]_{z_{n}=z_{n}^{0}}=0,(1 \leq n \leq N)$. With the dimensionless parameters $u_{n} \equiv z_{n}^{0} / \ell(n=1,2, \ldots, N)$, where $\ell \equiv \sqrt[3]{e^{2} / 4 \pi \epsilon_{0} M \omega_{z}^{2}}$ sets the scale of ion spacings in the linear crystal, this condition yields a set of equations $u_{j}-\sum_{n=1}^{j-1} 1 /\left(u_{j}-u_{n}\right)^{2}+\sum_{n=j+1}^{N} 1 /\left(u_{j}-u_{n}\right)^{2}=0$, which can be solved numerically to determine $z_{n}^{0}$ for any larger ion array [17]. To find all the phonon modes, one then just needs to expand the potential $V$ around the ions' equilibrium positions $\xi_{n}^{(0)} \equiv\left(x_{n}^{0}, y_{n}^{0}, z_{n}^{0}\right)$ with $\xi_{n}=\xi_{n}^{(0)}+q_{n}^{\xi}$. Under the harmonic approximation, we have $V=(1 / 2) \sum_{\xi, n, j} q_{n}^{\xi} q_{j}^{\xi}\left[\partial^{2} V / \partial \xi_{n} \partial \xi_{j}\right]_{\xi_{n}=\xi_{n}^{0}}=$ $\left(M \omega_{z}^{2} / 2\right) \sum_{\xi, n, j} A_{n j}^{\xi} q_{i}^{\xi} q_{j}^{\xi}$, where the matrix elements

$$
A_{n j}^{\xi}= \begin{cases}\beta_{\xi}^{2}+\sum_{p=1, p \neq j}^{N} a_{\xi} /\left|u_{j}-u_{p}\right|^{3} & (n=j) \\ -a_{\xi} /\left|u_{j}-u_{n}\right|^{3} & (n \neq j),\end{cases}
$$

with $\beta_{\xi}=\omega_{\xi} / \omega_{z}, a_{x}=a_{y}=-1$, and $a_{z}=2$ [19]. The eigenfrequencies $\omega_{\xi, k} \equiv \sqrt{\lambda_{\xi, k}} \omega_{\xi}$ and eigenvectors $\mathbf{b}_{j}^{\xi, k}$ of the normal phonon modes are obtained 
from diagonalization of the matrix $A^{\xi}=\left[A_{n j}^{\xi}\right]$ with $\sum_{n} A_{n j}^{\xi} \mathbf{b}_{n}^{\xi, k}=\lambda_{\xi, k} \mathbf{b}_{j}^{\xi, k}$. Then, with the substitution $q_{j}^{\xi}(t)=\sum_{k} \mathbf{b}_{j}^{\xi, k} \sqrt{\hbar / 2 M \omega_{\xi, k}}\left(a_{\xi, k}+a_{\xi, k}^{\dagger}\right)$, the motional Hamiltonian $H_{0}$ reduces to the standard form $H_{0}=$ $\sum_{\xi} \sum_{k=1}^{N} \hbar \omega_{\xi, k}\left(a_{\xi, k}^{\dagger} a_{\xi, k}+1 / 2\right)$, expressed by the annihilation and creation operators $a_{\xi, k}, a_{\xi, k}^{\dagger}$ of the $k$ th normal mode in the $\xi$ direction.

In order to visualize the TP and LP modes, we plot the complete mode spectrum for a 10-ion array in Fig. 1 (the modes along $x$ and $y$ directions are degenerate, so it is enough to show only the $x$-modes). We choose the trap frequency ratio $\beta_{x}=10$, which is typical for experiments and larger than the critical value of 5.3 to stabilize a linear configuration for $N=10$ ions. As opposed to the LP modes, the highest frequency TP mode is the centerof-mass mode at $\omega_{x}$. The frequency splitting between the CM mode and the second-to-highest mode (the bending mode) is about $0.05 \omega_{z}$ for $N=10$, which is significantly smaller than the splitting $(\sqrt{3}-1) \omega_{z}$ of the corresponding LP modes (the spectral structure of the TP modes is inverted compared to the LP modes, as seen in Fig. 1). For entangling local ions (such as neighboring ions), it is best to use the low-frequency TP "zigzag" mode [20] as it is more resolved from the other TP modes and most insensitive to the ion heating. But the CM mode has the advantage that it is equally coupled to all the ions, and thus more appropriate for gates between nonlocal ions (such as ions at different edges of the chain). When comparing features of gates using TP or LP modes, we parameterize the comparision of the $\mathrm{CM}$ modes for both cases.

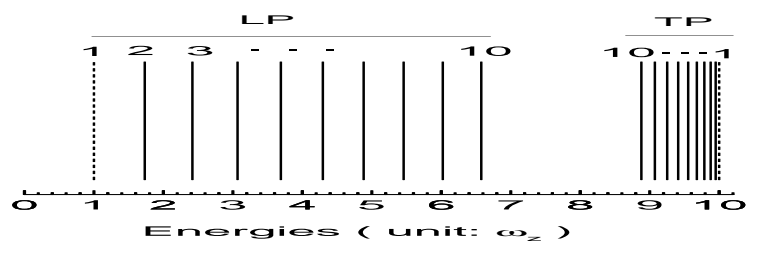

FIG. 1: The spectrum of the longitudinal (LP) and transverse phonon (TP) modes for a 10-ion array. The dashed lines (No. 1) denote the center of mass $(\mathrm{CM})$ mode.

General formalism of trapped ion quantum gates. First, we give a general formalism for multi-ion entangling quantum gates, which is valid with either TP or LP modes. The qubit for each ion is represented by two hyperfine states, denoted as $|0\rangle$ and $|1\rangle$ in general. The gate is achieved by applying a state-dependent acStark shift on the ions 11, 15], using two laser beams of equal intensity, wavevector difference $\Delta \mathbf{k}$ and frequency difference $\mu$. As it is common in experiments, we assume that the average ac-Stark shifts are the same for the $|0\rangle$ and $|1\rangle$ states for the ions in their equilibrium positions.
In this case, the Hamiltonian for the laser-ion interaction has the form

$$
H=\sum_{j=1}^{N} \hbar \Omega_{j} \cos \left(\Delta \mathbf{k} \cdot \mathbf{q}_{j}+\mu t\right) \sigma_{j}^{z}
$$

where $\Delta \mathbf{k} \cdot \mathbf{q}_{j}=\sum_{\xi} \Delta \mathbf{k}_{\xi} q_{j}^{\xi}(t)$, and $\Omega_{j}$ denotes the twophoton Rabi frequency of the $j$ th ion, which is proportional to the intensity of the driving laser. For convenience, $\Omega_{j}$ is assumed to be real, but it can be timedependent.

Now we assume that the relative wave vector $\Delta \mathbf{k}$ is chosen along a certain direction $\xi(\xi=x$ or $z)$, and motion in all modes in this direction is in the LambDicke regime $\eta_{\xi, k} \sqrt{\bar{n}_{\xi, k}+1} \ll 1$ for all $k$, where $\eta_{\xi, k}=$ $|\Delta \mathbf{k}| \sqrt{\hbar / 2 M \omega_{\xi, k}}$ is the Lamb-Dicke parameter and $\bar{n}_{\xi, k}$ the mean phonon occupation number of mode $(\xi, k)$. Note that for TP quantum gates $(\xi=x)$, the lower frequency LP modes $\xi=z$ (as well as the other transverse mode $\xi=y$ ) are decoupled and hence need not be confined within the Lamb-Dicke regime. To lowest order in $\eta_{\xi, k}$ and under the rotation-wave approximation, the interaction-picture Hamiltonian of the system is

$$
H_{I}=-\sum_{j, k=1}^{N} \hbar \chi_{j}^{\xi}(t) g_{\xi, j}^{k}\left(a_{\xi, k}^{\dagger} e^{i \omega_{\xi, k} t}+a_{\xi, k} e^{-i \omega_{\xi, k} t}\right) \sigma_{j}^{z},
$$

where the coupling constant $g_{\xi, j}^{k}=\eta_{\xi, k} \mathbf{b}_{j}^{\xi, k}$, and $\chi_{j}(t)=$ $\Omega_{j} \sin (\mu t)$ is proportional to the state-dependent force on the $j$-th ion.

The evolution operator corresponding to the Hamiltonian $H_{I}$ is given by 7,21 .

$$
U(\tau)=\exp \left[i \sum_{j} \phi_{j}^{\xi}(\tau) \sigma_{j}^{z}+i \sum_{j<n} \phi_{j n}^{\xi}(\tau) \sigma_{j}^{z} \sigma_{n}^{z}\right]
$$

where the displacement operator $\phi_{j}^{\xi}(\tau)=$ $\sum_{k}\left[\alpha_{\xi, j}^{k}(\tau) a_{\xi, k}^{\dagger}+\alpha_{\xi, j}^{k *}(\tau) a_{\xi, k}\right] \quad$ with $\quad \alpha_{\xi, j}^{k}(\tau)=$ $\int_{0}^{\tau} \chi_{j}(t) g_{\xi, j}^{k} e^{i \omega_{\xi, k} t} d t$, and the conditional phase $\phi_{j n}^{\xi}(\tau)=$ $2 \int_{0}^{\tau} d t_{2} \int_{0}^{t_{2}} d t_{1} \sum_{k} \chi_{j}\left(t_{2}\right) g_{\xi, j}^{k} g_{\xi, n}^{k} \chi_{n}\left(t_{1}\right) \sin \omega_{\xi, k}\left(t_{2}-t_{1}\right)$. In the case of a time-independent $\Omega_{j}, \alpha_{\xi, j}^{k}(\tau)$ and $\phi_{j n}^{\xi}(\tau)$ have the following explicit expressions:

$\alpha_{\xi, j}^{k}=\frac{\Omega_{j} g_{\xi, j}^{k}\left\{\mu+e^{i \omega_{\xi, k} \tau}\left[-\mu \cos (\mu \tau)+i \omega_{\xi, k} \sin (\mu \tau)\right]\right\}}{\left(\mu^{2}-\omega_{k}^{2}\right)}$

$\phi_{j n}^{\xi}=2 \Omega_{j} \Omega_{n} \sum_{k} \frac{g_{\xi, j}^{k} g_{\xi, n}^{k}}{\mu^{2}-\omega_{\xi, k}^{2}}\left\{\frac{\omega_{\xi, k}[-2 \mu \tau+\sin (2 \mu \tau)]}{4 \mu}+\right.$

$\left.\frac{\mu\left[\omega_{\xi, k} \cos \left(\omega_{\xi, k} \tau\right) \sin (\mu \tau)-\mu \cos (\mu \tau) \sin \left(\omega_{\xi, k} \tau\right)\right]}{\mu^{2}-\omega_{\xi, k}^{2}}\right\}$. 
To drive a conditional phase flip (CPF) gate between arbitrary ions $j$ and $n$, we take $\Omega_{j}$ to be nonzero only for these two ions, and set the laser detuning $\mu$ and the gate time $\tau$ so that $\phi_{j}^{\xi}(\tau)=\phi_{n}^{\xi}(\tau)=0$ and $\phi_{j n}^{\xi}(\tau)=\pi / 4$. In this case, the evolution operator $U(\tau)$ reduces to the $\mathrm{CPF}$ operator $U_{j n}=\exp \left(i \pi \sigma_{j}^{z} \sigma_{n}^{z} / 4\right)$.

TP versus LP quantum gates. To compare quantum gates based on TP vs. LP modes, let us start with the assumption that the driving laser can address individual phonon modes through frequency selection (resolvedsideband addressing). This requires the gate time $\tau$ to be much larger than $\tau_{\Delta} \equiv 2 \pi / \Delta$, where $\Delta$ is the characteristic frequency splitting of the phonon modes (we will see later that only one phonon mode dominates when $\left.\tau \geq 2 \tau_{\Delta}\right)$. The sideband addressing assumption, although not essential, allows us to derive simple analytic relations that permit a direct comparison between TP and LP gates.

With sideband addressing, we dominantly excite a particular phonon mode $(\xi, p)$ with frequency $\omega_{\xi, p}$ by adjusting the laser detuning $\mu$ close to $\omega_{\xi, p}$ with $\mu=$ $\omega_{\xi, p}+2 \pi l_{\xi} / \tau$, where $l_{\xi}$ is an integer, typically chosen as 1 or $-1[3,7]$. The qubit state and the motion state should be disentangled at the end of the gate, which requires $\phi_{j}^{\xi}(\tau)=\phi_{n}^{\xi}(\tau)=0$ and thus $\tau=l_{\xi}^{\prime} \pi / \omega_{\xi, p}$ (see Eq. (4)), where $l_{\xi}^{\prime}$ is another integer (typically, $l_{\xi}^{\prime} \gg l_{\xi}$ ). From Eq. (6), the conditional phase shift is found to be

$$
\phi_{j n}^{\xi}(\tau)=-\frac{\mathbf{b}_{\xi, j}^{p} \mathbf{b}_{\xi, n}^{p}}{4 \pi} \frac{\eta_{\xi, p}^{2} \Omega^{2} \tau^{2}}{1+l_{\xi} / l_{\xi}^{\prime}} .
$$

The condition $\phi_{j n}^{\xi}(\tau)=\pi / 4$ can be satisfied with an appropriate choice of the Rabi frequency $\Omega$.

To consider inherent infidelity of the gate operation, we note that all the above results are derived based on the Lamb-Dicke condition. In practice, the Lamb-Dicke parameter is finite, and the thermal motion of the ions will induce some fluctuation of the Rabi frequency $\Omega^{2}$ in Eq. (6) and lead to gate errors. To estimate this noise, we need to expand the Hamiltonian of Eq. (2) to higherorders in the Lamb-Dicke parameters. The effect of these higher-order terms is to replace $\Omega$ in Eq. (6) with an effective Rabi frequency $\Omega_{n}^{\xi}$ that depends on the phonon number $n_{\xi}$ of the ion vibrational mode $(\xi, p)$. To the next order of the Lamb-Dicke parameter $\eta_{\xi, p}$, we find $\left(\Omega_{n}^{\xi}\right)^{2} \approx$ $\Omega_{\xi}^{2}\left[1-\eta_{\xi, p}^{2}\left(2 n_{\xi}+1\right)\right][\underline{3}]$. The gate fidelity $F_{g}^{\xi}[22]$ is then found to be $F_{g}^{\xi}=\frac{1}{2}+\frac{1}{2} \sum_{n=0}^{\infty} P_{n}^{\xi} \cos \left[\frac{\pi}{2} \eta_{\xi, p}^{2}(2 n+1)\right][23]$. When the initial phonon number distribution $P_{n}^{\xi}$ takes the form of a thermal state, $P_{n}^{\xi}=\bar{n}_{\xi}^{n} /\left(1+\bar{n}_{\xi}\right)^{n+1}$, we find to lowest order in $\eta_{\xi, p}$ a gate infidelity $F_{i n}^{\xi} \equiv 1-F_{g}^{\xi}$ of

$$
F_{i n}^{\xi} \approx \pi^{2} \eta_{\xi, p}^{4}\left(\bar{n}_{\xi}^{2}+\bar{n}_{\xi}+1 / 8\right) .
$$

As the TP mode has a larger vibrational frequency, the TP quantum gates have a significantly smaller gate infidelity from thermal ion motion. Even if we assume the
TP and the LP modes have the same mean phonon number $\bar{n}_{x}=\bar{n}_{z}$, the infidelity for the TP gate is smaller by a factor of $F_{i n}^{x} / F_{i n}^{z}=\eta_{x, p}^{4} / \eta_{z, p}^{4}=\left(\omega_{z, p} / \omega_{x, p}\right)^{2}$. In practice, if the TP and LP modes are subject to the same heating mechanism, and initially prepared with the same laser cooling technique, we further expect $\bar{n}_{x} \ll \bar{n}_{z}$ due to differences in the initial temperature and the heating rate of TP vs. LP modes. The temperature limit $T_{L}$ can be considered as independent of the phonon frequency $\omega_{\xi, p}$ for Doppler cooling, and is roughly proportional to $1 / \omega_{\xi, p}$ for the Raman sideband cooling. So the contribution of $T_{L}$ to the mean phonon number $\bar{n}_{\xi}$, estimated as $k_{B} T_{L} / \hbar \omega_{\xi, p}$, is taken to be $\left(1 / \omega_{\xi, p}\right)^{\gamma}$, where $\gamma$ is between 1 and 2 . The ion heating rate $\dot{\bar{n}}_{\xi}$ for the phonon mode $(\xi, p)$ is proportional to the noise power spectrum $S\left(\omega_{\xi, p}\right)$ at the frequency $\omega_{\xi, p}$ 24], taken to be independent of frequency (white noise) or proportional to $1 / \omega_{\xi, p}(1 / f$ noise $)$. For these practical noise sources 24], the average phonon occupation number therefore scales as $\left(1 / \omega_{\xi, p}\right)^{\gamma^{\prime}}$, with $\gamma^{\prime}$ again between 1 and 2 . If we assume the term $\bar{n}_{\xi}^{2}$ dominates in the infidelity expression (8), which is likely for many ions in a crystal, the infidelity ratio of TP vs. LP gates is then $F_{i n}^{x} / F_{i n}^{z} \sim \bar{n}_{x}^{2} \eta_{x, p}^{4} /\left(\bar{n}_{z}^{2} \eta_{z, p}^{4}\right) \sim\left(\omega_{z, p} / \omega_{x, p}\right)^{2+\gamma+\gamma^{\prime}}$, where we have assumed that the gate time $\tau$ is the same for both cases. For the CM modes, $\omega_{z, p} / \omega_{x, p}$ is given by the trap frequency ratio $\beta_{x}=\omega_{z} / \omega_{x}$. So, compared with LP gate, the inherent infidelity of the TP gate could be reduced by a factor of $\beta_{x}^{4}$ to $\beta_{x}^{6}$, which is significant even for a moderate trap frequency ratio of $\beta_{x} \sim 5$.

Now we look at the cost of the TP quantum gate. As the TP modes have a higher vibrational frequency, it is harder to excite them, and we need more laser intensity for the same gate speed. From Eq. (7), to have the same gate time $\tau$, the ratio of the required laser intensity $I_{x} / I_{z}$ (note that $I_{\xi}$ is proportional to the two-photon Rabi frequency $\left.\Omega_{\xi}\right)$ is given by $I_{x} / I_{z} \sim \eta_{z, p} / \eta_{x, p}=\sqrt{\omega_{x, p} / \omega_{z, p}}=$ $\sqrt{\beta_{x}}$ (we neglect $l_{\xi} / l_{\xi}^{\prime}$ in Eq. (7) as it is typically much less than 1). So, although we need additional laser power for the TP quantum gate, this cost is moderate compared with the improvement we achieve in the gate fidelity. If we take into account of different laser excitation configurations for the TP and the LP gates, this cost is even less. For the LP gate, the relative wave vector $\Delta \mathbf{k}$ of the Raman laser beams needs to be along the trap axis, but one cannot directly apply lasers in that direction, so in practice both of the laser beams have a $45^{\circ}$ to the trap axis 11, 15. However, for the TP quantum gate, one can apply counter-propagating laser beams along the $x$-axis so that $\Delta \mathbf{k}$ is perpendicular to the ion string. Because of this difference, the laser intensity ratio $I_{x} / I_{z}$ is actually $\sqrt{\beta_{x} / 2}$ instead of $\sqrt{\beta_{x}}$. The above laser configuration difference also gives some practical advantages for the TP quantum gates: first, as the laser beams are perpendicular to the ion string, it is easy to have the 
same relative laser phase for different ions, as assumed in the gates above. For LP gates, it is difficult to achieve such a condition for different ions as they are not equally spaced. It usually requires subtle control of the ion distance through adjustment of the trap frequency [10, 11], and such a technique is not scalable to many ions. Second, with the transverse focused laser beams, it is also easier to achieve separate addressing of different ions. For the TP gate, one does not need to have a large longitudinal $\omega_{z}$ to achieve the Lamb-Dicke condition, so the ion distance is not limited, and one can combine separate addressing with a many-ion setup, which is desired for scalable quantum computation.

Arbitrary-speed TP gates through minimal control of the laser beams. As we have mentioned before, the TP modes have small frequency splittings, so resolving a particular TP mode could be difficult for a large ion array. If we want to achieve a high-speed gate, it is necessary to go beyond the sideband addressing (single-mode) limit. Fortunately, for any practical qubit number (up to a few hundreds, for instance), it is always possible to take into account of all the phonon modes and design high-fidelity gates with no limitation to the gate speed $[5,6,7]$. These fast gates are in general based on control of the laser pulses.

Here, similar to Ref. [7], we use a simple sequence of laser pulses which take minimum experimental control. We chop a continuous-wave laser beam into $m$ equal-time segments (through acoustic/electric modulator, for instance), with a constant but controllable Rabi frequency $\Omega_{p}$ for the $p$ th $(p=1,2, \cdots, m)$ segment. The state-dependent force $\chi(t)$ in the Hamiltonian (3) then takes the form $\chi(t)=\Omega_{p} \sin (\mu t)$ for the time interval $(p-1) \tau / m \leq t<p \tau / m$ (For simplicity of the notation, we omit the direction index $\xi$ in the following and take $\xi=x$ by default). With a large number of ions but a small number of control parameters $\Omega_{p}$, the displacements $\alpha_{j}^{k}(\tau)$ (and thus $\phi_{j}(\tau)$ ) in the evolution operator (4) may not exactly reduce to zero. But as long as they are small, we still can get a high-fidelity gate. In this case, for a gate on the ion-pair $(j, n)$ in an $N$-ion array, the gate infidelity due to the residue small displacements $\alpha_{j}^{k}$ is given by

$$
F_{i n} \approx \sum_{k} \bar{\beta}_{k}\left[\left|\alpha_{j}^{k}\right|^{2}+\left|\alpha_{n}^{k}\right|^{2}\right] / 4,
$$

where $\bar{\beta}_{k}=\operatorname{coth}\left[\sqrt{\mu_{k} / 4} \ln n\left(1+1 / \bar{n}_{1}\right)\right]$ with $\bar{n}_{1}$ representing the mean phonon number of the CM TP mode. For the above expression, we have assumed that the TP modes are in their thermal equilibrium state. The task of control is to get a small infidelity $F_{i n}$ by choosing an optimal laser detuning $\mu$ and a minimum number of the control parameters $\Omega_{p}$.

First, let us consider the gate with a single continuouswave laser beam (the number of segments $m=1$ ). The

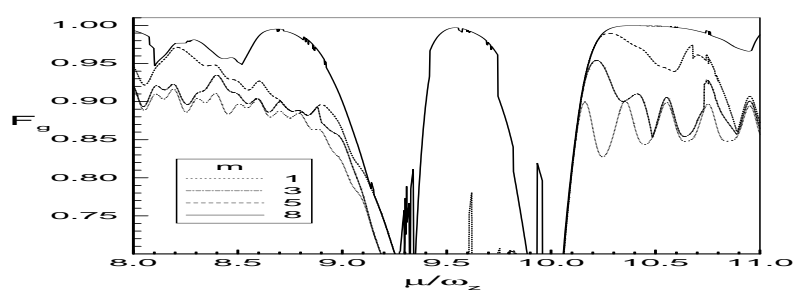

FIG. 2: For the two edge ions in a 10-ion array, the TP gate fidelity as a function of the detuning $\mu$ with the gate time $\tau=5 \tau_{0}, \bar{n}_{1}=3, \beta_{x}=10$, and the number of segments $m=1,3,5,8$, respectively.

only control parameter is the detuning $\mu$. We find that for the gate on two edge ions (1st and 2nd ions) in a 10ion array, as long as the gate time $\tau \geq 37 \tau_{0}$, where $\tau_{0} \equiv$ $2 \pi / \omega_{z}$, the gate infidelity $F_{\text {in }} \leq 0.99 \%$. For this and the following calculations, we take $\bar{n}_{1} \approx 3$ which corresponds to a pretty high temperature. The optimal $\mu$ is very close to the value $\omega_{x}+2 \pi / \tau$. Note that a gate with $\tau \approx 37 \tau_{0}$ has been faster than any ion gate implemented so far in the lab [11, 15]. For this gate, the time $\tau$ is close to $\tau_{\Delta} \equiv$ $2 \pi / \Delta\left(\tau \approx 1.8 \tau_{\Delta}\right)$, where $\Delta$ is the frequency splitting between the CM TP mode and the bending mode. So, besides the dominant CM mode, various TP modes are indeed slightly excited during the gate and contribute to the conditional phase $\phi_{j n}(\tau)$. But with the optimal $\mu$, all these modes evolve along an almost-closed loop in the phase space $\left(\alpha_{i}^{k} \approx 0\right.$, although not exactly), so we still have a high fidelity gate. The required Rabi frequency from the exact numerical calculation is very close to the one given by the analytic formula (7) from the singlemode approximation (within a $6 \%$ error).

If we further increase the gate speed with $\tau<37 \tau_{0}$, the gate fidelity quickly decreases, so we need to chop the continuous-wave laser beam into more segments with $m>1$ to increase the fidelity. With a sufficiently large $m$, a high-fidelity gate with an arbitrary gate speed can be achieved. In Fig.2, we show the calculation result for the gate time $\tau=5 \tau_{0}$. With the number of segments $m=1,3,5,8$, the gate infidelity is given by $10 \%, 4.9 \%, 1.0 \%, 0.1 \%$, respectively, with the optimized parameters $\mu$ and $\Omega_{1}, \Omega_{2}, \cdots, \Omega_{m}$. We also calculate the gate infidelity for other ion-pairs, and the results are qualitatively similar. For instance, with $\tau=5 \tau_{0}$ and the number of segments $m=1,3,5,8$, respectively, the gate infidelity $F_{\text {in }}$ is given by $5.5 \%, 1.8 \%, 0.22 \%$, $0.07 \%$ for the two center ions ( 5 th and 6 th ions), and by $40 \%, 25 \%, 8.5 \%, 0.99 \%$ for the 1 st and 10 th ions at the far ends of the string (the worst case).

In summary, we have proposed to use the transverse phonon mode to achieve quantum gates, and shown that these TP gates are much less sensitive to ion heating and thermal motion compared with conventional gates based on the longitudinal modes. We also show how to achieve 
arbitrary-speed TP quantum gates based on control of the laser pulses.

This work is supported by the NSA and the DTO under ARO contract W911NF-04-1-0234, the NSF under grant 0431476, and the A. P. Sloan Foundation. S.L.Z. also acknowledges support by the NCET.

[1] For a review, see C. Monroe, Nature (London) 416, 238 (2002).

[2] J. I. Cirac and P. Zoller, Phys. Rev. Lett. 74, 4091 (1995); Nature 404, 579 (2000).

[3] A. Sorensen and K. Molmer, Phys. Rev. Lett. 82, 1971 (1999); Phys. Rev. A 62, 022311 (2000).

[4] G. J. Milburn, S. Schneider, and D. F. V. James, Fortschr. Phys. 48, 801 (2000).

[5] J. J. Garcia-Ripoll, P. Zoller, and J. I. Cirac, Phys. Rev. Lett. 91, 157901 (2003); Phys. Rev. A 71, 062309 (2005).

[6] L.-M. Duan, Phys. Rev. Lett. 93, 100502 (2004).

[7] S. -L. Zhu, C. Monroe, and L. -M. Duan, Europhys. Lett. (in press, 2006); quant-ph/0508037

[8] D. J. Wineland et al., J. Res. Natl. Inst. Stand. Technol. 103, 259 (1998).

[9] C. Monroe et al., Phys. Rev. Lett. 75, 4011 (1995).
[10] C. A. Sackett et al., Nature (London) 404, 256 (2000).

[11] D. Liebfried et al., Nature (London) 422, 412 (2003).

[12] H. Haffner et al., ibid. 438, 643 (2005); S. Gulde et al., Nature (London) 421, 48 (2003).

[13] B. B. Blinov et al., Nature (London) 428, 153 (2004); L.-M. Duan et al., Quantum Inf. Comput. 4, 165 (2004).

[14] D. Leibfried et al., ibid. 438, 639 (2005); M. D. Barrett et al., Nature (London) 429, 737 (2004).

[15] K.-A. Brickman et al., Phys. Rev. A 72050306 (2005).

[16] D. Stick et al., Nature Physics 2, 36 (2006).

[17] D. F. V. James, Appl. Phys. B: Lasers Opt. 66, 181 (1998).

[18] A. Steane, Appl. Phys. B 64, 623 (1997).

[19] X.-L. Deng, D. Porras, J. I. Cirac, quant-ph/0509197

[20] J. P. Schiffer, Phys. Rev. Lett. 70,818 (1993).

[21] S. -L. Zhu and Z. D. Wang, Phys. Rev. Lett. 91, 187902 (2003).

[22] The gate fidelity is defined as $F_{g}^{\xi}=\left\langle\Psi_{0}\left|U_{i j}^{\dagger} \rho_{r} U_{i j}\right| \Psi_{0}\right\rangle$ with a typical initial state $\left|\Psi_{0}\right\rangle=\left(|0\rangle_{i}+\right.$ $\left.|1\rangle_{j}\right) \otimes\left(|0\rangle_{j}+|1\rangle_{j}\right) / 2$. The density operator $\rho_{r}=\operatorname{Tr}_{m}\left[U_{\xi}(\tau)\left|\Psi_{0}\right\rangle\left\langle\Psi_{0}\right| U_{\xi}^{\dagger}(\tau)\right]$, where the trace is over the motional states of all the ions.

[23] For this calculation, the gate time $\tau$ remains the same as it is fixed by the condition $\phi_{j}^{\xi}(\tau)=\phi_{n}^{\xi}(\tau)=0$.

[24] Q. A. Turchette, et al., Phys. Rev. A 61, 063418 (2000); L. Deslauriers, et al., Phys. Rev. A 70, 043408 (2004). 